\title{
A Study of the Cyclotron Gas-Stopping Concept for the Production of Rare Isotope Beams
}

\author{
M. Sternberg, G. Savard \\ Argonne National Laboratory and The University of Chicago
}

October 26, 2018

\begin{abstract}
The proposed cyclotron gas-stopping scheme for the efficient thermalization of intense rare isotope beams is examined. Simulations expand on previous studies and expose many complications of such an apparatus arising from physical effects not accounted for properly in previous work. The previously proposed cyclotron gas-stopper geometry is found to have a near null efficiency, but extended simulations suggest that a device with a much larger pole gap could achieve a stopping efficiency approaching roughly $90 \%$ and at least a 10 times larger acceptance. However, some of the advantages that were incorrectly predicted in previous simulations for high intensity operation of this device are compromised.
\end{abstract}

\section{Introduction}

The thermalization of energetic beams produced by fragmentation, in-flight fission or fusion-evaporation reactions is an important technology for the production of low-energy rare isotope beams. It can provide access to radioactive beams of refractory or reactive elements not amenable to the ISOL technique. These thermalizing techniques are currently being aggressively developed and are essential to the next generation of radioactive beam experiments. The production of such beams would allow reaccelerated beam experiments to venture into a regime of exotic isotopes beyond the reach of current target/ion source assemblies, allowing for a wealth of new experiments to be developed.

Thermalizing energetic ions in a gas volume and extracting them for postacceleration is a difficult task, but one that has now been realized in many facilities interested in ISOL type experiments [1, 2, 3, 4. Such facilities typically cool beams through energy loss in high-purity helium, guide the thermalized ions to a nozzle where they are carried by gas flow through a differential pumping aperture after which the heavy ions are extracted and transported to a postaccelerator or used in experiments involving cooled rare isotopes. While this approach has been shown to work well at low intensity, most approaches have shown limitations with incident beam intensity many orders of magnitude lower 
than what is expected for next generation radioactive beam facilities [5, 6]. The cyclotron stopper has been suggested as a way to overcome this limitation.

\section{Proposed Concept}

The cyclotron stopper technique was proposed by Katayama et al. [7] and a detailed study by Bollen et al. 8] was recently published. This approach involves the use of a weakly focusing cyclotron magnet which provides ions an essentially unlimited path length due to cyclotron motion, allowing the use of gas pressures significantly lower than current techniques. As the reaction products ionize the helium gas they slow down and spiral in towards the center where they eventually reach thermal energies. The desired end effect is to concentrate cooled heavy ions near the center of the magnet, away from the bulk of the ionized helium.

The separation of cooled heavy ions from the majority of ionized gas allows for efficient extraction and removes several difficulties that have plagued most other gas catcher schemes. The undesirable bulk of helium ions can then be removed with the use of charge collection electrodes without affecting the cooled fragments. Near the center of the magnet, the thermalized heavy ions are carried towards an RFQ ion guide through the use of a DC electric field, an RF-carpet, and gas flow.

The main features that make such a concept attractive for use in stopping energetic ions are its extremely long stopping length, low gas pressure, and the clear separation between ionized helium and cooled heavy ions. A study of this concept was reported in Ref. [8] and has been used as a starting point for this study. Fig. 1 illustrates a rough layout for the concept proposed in Ref. 8. Bollen et al. conclude that for a prototypical Bromine radioactive beam a stopping efficiency approaching $100 \%$ can be reached in such a device with a $4 \mathrm{~cm}$ gap between the charge collection electrodes. This result is surprising in that the lateral straggling of such a beam coming to rest in gas, in the absence of magnetic or electric fields, far exceeds this dimension.

We therefore repeated the simulations while including various effects that were either ignored or improperly treated in Ref. [8]. In addition, we also performed simulations for injection of beam with phase-space more representative of modern large acceptance spectrometers. For the geometry and beam properties used in Ref. [8, we find a stopping efficiency down by over two orders of magnitude compared to their results. Our simulations indicate that a pole gap up to 18 times larger is required to obtain sizable efficiencies. This not only increases the technical difficulties with the magnet but also removes some of the advantages that were proposed for operation at high intensity. By modifying the injection scheme we can, in addition, increase the acceptance of the device by roughly an order of magnitude.

The approach taken below was to first reproduce the results of Ref. [8], then systematically improve on this simulation by:

- Correcting the charge-changing collision method used in that paper. 


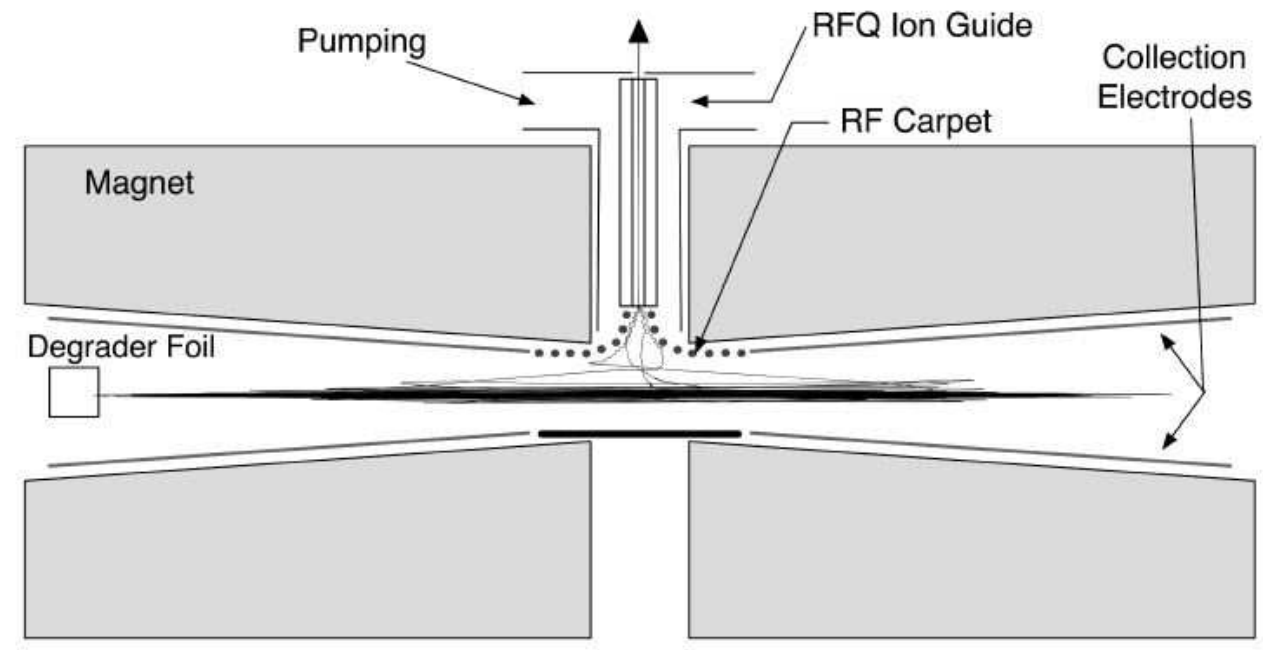

Figure 1: Concept for the cyclotron gas-stopper studied in Ref. [8].

- Including proper charge state of ions in solids for beam exiting the degrader.

- Adding angular straggling.

- Performing simulations with larger phase-space for the incoming beam.

- Investigating alternative means of focusing.

The basis for the changes between the present simulations and the work of Ref. 8 is given when each change is made and conclusions are stated on the status of this approach as an alternative for high intensity operation for both the original and the new larger acceptance geometry proposed here.

\section{Simulation}

\subsection{Basis For Simulation}

All of the simulation details discussed in this subsection, unless otherwise stated, have been taken directly from Ref. [8] and have been used as a starting point for simulations discussed later in this paper. Information and details beyond what are discussed here can be found in Ref $[8$. The simulations are performed using a Monte Carlo ray tracing code and consist of a weakly focusing cyclotron magnet, $1 \mathrm{~m}$ in radius, filled with 10 mbar helium gas at zero degrees Celsius. The peak magnetic field of the magnet is $B_{o}=2 \mathrm{~T}$ with a field index of $n_{o}=$ 0.2 and a radius of injection $r_{\mathrm{inj}}=0.8 \mathrm{~m}$. 
Bromine is used as a test case for the simulation because of its moderate mass and because of the charge-exchange data available for this element. The test beam consists of $100 \mathrm{MeV} / \mathrm{u}{ }^{78} \mathrm{Br}$ passing through an aluminum degrader resulting in a beam with an average energy of $610 \mathrm{MeV}$. The initial simulation assumes that the beam exiting the degrader has an average energy spread $\Delta \mathrm{E} / \mathrm{E}$ of $20 \%$, a beam half width of $5 \mathrm{~mm}$ and a beam half divergence of $10 \mathrm{mrad}$.

The motion is modeled for non-relativistic ions using an axially symmetric form $\vec{B}(\vec{\rho}, z)=B_{\rho} \cdot \hat{\rho}+B_{z} \cdot \hat{z}$. Where, in a paraxial approximation, $B_{\rho}$ and $B_{z}$ are given by:

$$
\begin{gathered}
B_{\rho}=-\left(n_{o} \cdot B_{o} / r_{\mathrm{inj}}\right) z \\
B_{z}=B_{o}-\left(n_{o} \cdot B_{o} / r_{\mathrm{inj}}\right) \rho
\end{gathered}
$$

Energy loss is modeled using stopping tables for bromine ions in helium provided by SRIM [9] for a range of energies from $1 \mathrm{GeV}$ to $100 \mathrm{eV}$. Energy straggling is not considered since it corresponds to a deviation in the total path length of less than $5 \%$ and therefore does not have a sizable impact on the simulation. Angular straggling was also not considered in the original simulation because its effects were thought by 8 to be small. Verifying this assumption was one of the leading motivations for trying to reproduce the simulations reported in Ref. [8]. As will be evident later, the effects due to angular straggling are not negligible.

Charge-exchange collisions of $\mathrm{Br}$ with helium are modeled for single-electron exchange. Multiple-electron loss and capture can be ignored since their crosssections are in general orders of magnitudes smaller. The average charge state $\bar{q}$ as a function of ion velocity $v$ is calculated according to semi-empirical formulae fitted to experimental data [10],

$$
\begin{array}{cc}
\bar{q} / Z=\frac{\lg \left(v / m_{1} Z^{\alpha_{1}}\right)}{\lg \left(n_{1} Z^{\alpha_{2}}\right)} & (0.3 \leq \bar{q} / Z \leq 0.9) \\
\bar{q} / Z=A v Z^{-1 / 2} & (\bar{q} / Z<0.3)
\end{array}
$$

where $Z$ is the proton number and $m_{1}, n_{1}, \alpha_{1}, \alpha_{2}$, and $A$ are parameters fitted to experimental data.

The cross-sections for charge loss $\sigma_{l}$ and capture $\sigma_{c}$ for a given charge state $q$ are given by:

$$
\begin{gathered}
\sigma_{c}=\frac{\sigma_{o}}{2} \cdot e^{a_{c}(q-\bar{q})} \\
\sigma_{l}=\frac{\sigma_{o}}{2} \cdot e^{a_{l}(\bar{q}-q)}
\end{gathered}
$$

$\sigma_{o}, a_{c}$, and $a_{l}$ are parameters whose energy dependence have been parameterized by fitting Eqns. 5 \& 6 to experimental data for $\mathrm{Br}$ in helium at a range of energies up to $14 \mathrm{MeV}$ obtained from a review article by Betz et al. [10]. $\sigma_{o}$ strongly determines the total cross section $\sigma$ for a charge exchange collision given 
by $\sigma=\sigma_{c}+\sigma_{l}$. Similarly, $a_{l}$ and $a_{c}$ determine the width of the charge state distribution for a given $\bar{q}$. The ratio of $\sigma_{c}$ and $\sigma_{l}$ to $\sigma$ give the probability for charge capture and charge loss, respectively, during a charge-exchange collision.

Beyond $14 \mathrm{MeV}$ the values for $a_{l}$ and $a_{c}$ are fixed. This is not of much concern since the width of the charge distribution does not vary greatly over a broad range of energies and, in addition, small variations in the width of the charge distribution do not have a significant effect on simulations. However, it is necessary to know $\sigma_{o}$ or more importantly $\sigma$ for energies greater than $14 \mathrm{MeV}$, since $\sigma$ directly determines the mean free path $\lambda=1 /(n \cdot \sigma)$, where $\mathrm{n}$ is the number density of the gas. If $\lambda$ is too large relative to the radius of cyclotron motion, it will result in dramatic changes in trajectory as an ion moves from one charge state to another. If instead lambda is relatively small, the ions trajectory will be "smoothed out" over the course of several charge exchange collisions, resulting in an overall trajectory corresponding to the average charge state $\bar{q}$.

The magnitude of $\sigma_{o}$ is determined for energies above $14 \mathrm{MeV}$ and up to $700 \mathrm{MeV}$ using Bohr's expressions $\sigma_{c_{-} \text {Bohr }}$ and $\sigma_{l_{-} \text {Bohr }}$ for single-electron capture and loss by heavy ions in light targets given by Eqns. $4.5 \& 4.6$ of Ref. [10].

$$
\begin{aligned}
& \sigma_{c_{-} \text {Bohr }} \sim 4 \pi a_{o}^{2} Z^{1 / 3} Z_{T}^{5}\left(v_{o} / v\right)^{6} \\
& \sigma_{l_{-} \text {Bohr }} \sim 4 \pi a_{o}^{2} Z^{1 / 3} Z_{T}^{2}\left(v_{o} / v\right)^{3}
\end{aligned}
$$

Here, $v$ is the ion velocity, $a_{o}$ is the Bohr radius $a_{o}=5.292 \cdot 10^{-9} \mathrm{~cm}, v_{o}$ is the Bohr velocity $v_{o}=2.118 \cdot 10^{8} \mathrm{~cm} / \mathrm{s}, Z$ is the atomic number of the projectile nucleus, and $Z_{T}$ is the atomic number of the gas target. $\sigma_{o}$, determined in this way, combined with $\bar{q}, a_{l}$ and $a_{c}$, calculated as previously stated, are used in Eqns. 5 \& 6 to give the values for $\sigma_{c}$ and $\sigma_{l}$ that are used to determine the probabilities for charge exchange in the simulation. This is different from what was stated in Ref. [8], where they claimed to use Eq. 4.7 of Ref. [10].

$$
\sigma_{l_{-} \text {Bohr }} \sim \sigma_{c_{-} \text {Bohr }} \sim \pi a_{o}^{2}\left(Z^{1 / 3}+Z_{T}^{1 / 3}\right)\left(v_{o} / v\right)^{2}
$$

Contrary to what is stated in Ref. [8, Eq. 9 is, as stated in Ref. [10, an equation for heavy ions in heavy targets. For $610 \mathrm{MeV}$ bromine the use of Eq. 9 in calculating $\sigma$ results in a nearly 3 fold increase as compared to calculations using Eqns. 8 \& 7 In addition, it is reported in Ref. 8 that $610 \mathrm{MeV}$ bromine in 10 mbar of helium has a mean free path of $6 \mathrm{~mm}$. However, 10 mbar of 273 $\mathrm{K}$ helium $\left(n=2.6 \cdot 10^{17}\right.$ atoms $\left./ \mathrm{cm}^{3}\right)$ with a mean free path of about $6 \mathrm{~mm}$ corresponds to a cross-section for charge-exchange of nearly $\sigma=7 \cdot 10^{-18} \mathrm{~cm}^{2}$. This is roughly 3 times larger than the value of $\sigma$ calculated using Eq. 9 and about 9 times the value of $\sigma$ given by the more appropriate Eqns. $8 \& 7$. We found no way of reconciling these differences.

Furthermore, experimental values for charge-exchange cross-sections provide an upper limit on reasonably acceptable values. The charge-exchange crosssection for the mean charge state of $8.5 \mathrm{MeV} / \mathrm{u} \mathrm{Kr}^{+27}$ in $\mathrm{N}_{2}$ is measured to be $\sigma \approx 4 \cdot 10^{-18} \mathrm{~cm}^{2}[12$. In helium this cross-section would be lower by a factor 
of two in order to account for the diatomic nature of $\mathrm{N}_{2}$ and an additional factor of at least $\left(Z_{\mathrm{N}} / Z_{\mathrm{He}}\right)^{2} \approx 10$ in accordance with Eqns. 8 \& 7 A similar measurement [13] reports a value of $\sigma \approx 2 \cdot 10^{-20} \mathrm{~cm}^{2}$ for $8.55 \mathrm{MeV} / \mathrm{u} \mathrm{V} \mathrm{V}^{+21}$ in He. Both of these measurements support the smaller values of $\sigma$ calculated using Eqns. 8 \& 7 (actually, this data points to even smaller values of $\sigma$ than what is predicted by Eqns. 8 \& 7 at these energies).

\subsection{Reproducing Previous Simulation}

Simulations have been produced exactly as described above using SIMION ray tracing software [11 in an attempt to reproduce the results reported by Ref. 8. without any angular straggling from ion-gas collisions. In order to match the parameters used by Ref. [8, $\lambda$ was limited to values less than $6 \mathrm{~mm}$, a value substantially smaller than those suggested by Eqns. 8 \& 7 or data from experimental charge-exchange cross-sections. While the results of these simulations appear similar to those reported in Ref. 8], the orbits are less regular, a small percentage of ions are lost due to collisions with the degrader as they cycle through the first few cyclotron orbits, and the final ion position distribution does not form a distinct toroidal stopping volume. This is due to the fact that the trajectories of the ions are not completely "smoothed" out from charge-exchange collisions, which is clearly visible when the trajectories from this simulation presented in Fig. 2 are compared directly to Fig. 3 where, to better reproduce the results of their simulations, $\lambda$ was arbitrarily further limited to values below $0.2 \mathrm{~mm}$ (corresponding to $\sigma_{o}>2 \cdot 10^{-16} \mathrm{~cm}^{2}$ ).

The trajectories of Fig. 3] are in perfect agreement with Ref. [8], where the only appreciable differences in ion trajectories are due to the momentum and angular spread of ions exiting the degrader. Furthermore, the maximum density of deposited energy per ion is found to be about $125 \mathrm{keV} / \mathrm{cm}^{2}$ with a clear separation between the bulk of ionization and the $30 \mathrm{~cm}$ radius toroidal stopping volume with a $3 \mathrm{~cm}$ height, again, this is in perfect agreement with Ref. 8. As previously predicted, such a configuration should be able, if these simulations were correct, to quickly remove the bulk of ionized helium ions across the $4 \mathrm{~cm}$ electrode gap fast enough to support beam rates as high as $10^{8} / \mathrm{s}$.

\subsection{Necessary Improvements}

The simulations discussed in the previous subsection have not addressed several unavoidable problems. Thus far, the mean free path for charge-exchange collisions has been artificially limited to small values. It is necessary to use cross-sections that agree with experimental results as well as theory, such as cross-sections calculated using Eqns. 8 \& 7 In addition, the path length between charge-exchange collisions is assumed to be the same as the mean free path. This is not realistic and fails to accommodate the fact that the path length should vary about the mean free path. A more appropriate method is to 


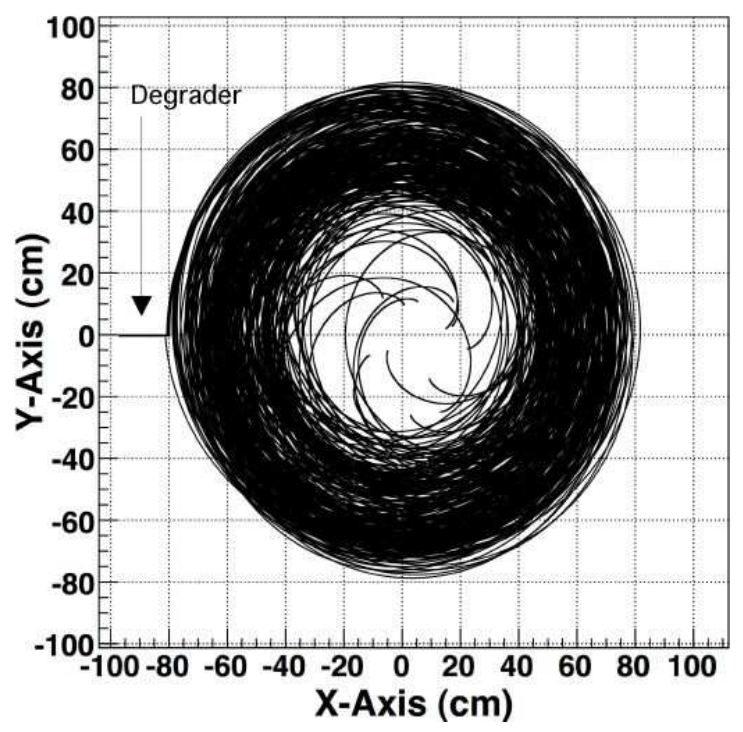

Figure 2: Trajectories of 15 ions projected onto the x-y plane with $\lambda$ limited to values less than $6 \mathrm{~mm}$ as used in Ref. [8]. The degrader foil is located on the $\mathrm{x}$-axis at $\mathrm{x} \leq-79.5 \mathrm{~cm}$.

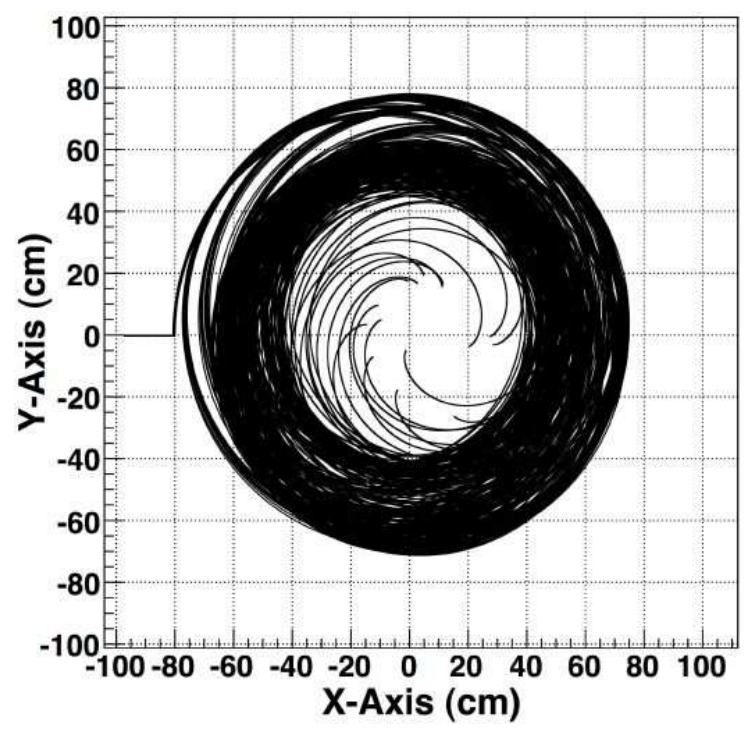

Figure 3: Trajectories of 15 ions projected onto the x-y plane with $\lambda$ limited to values less than $0.2 \mathrm{~mm}$. The degrader foil is located on the $\mathrm{x}$-axis at $\mathrm{x} \leq-79.5$ $\mathrm{cm}$. 
sample the path length $l$ using the following formula:

$$
l=-\lambda \cdot \ln p
$$

Where $\lambda$ is the mean free path between charge-exchange collisions discussed earlier and $p$ is a random number between 0 and 1 .

Fig. 2 demonstrates that in the regime where $\lambda$ is larger than $6 \mathrm{~mm}$ the effects on the ion trajectories due to charge-exchange collisions are not "smoothed out". It is necessary, therefore, to recognize that ions exiting a solid degrader will have a mean charge state higher than the mean charge state for the same ions in a gas. This will have an appreciable effect if ions travel a sizable fraction of an orbit before returning to the mean charge state. The average charge state for ions in a solid can be calculated using Eq. 3 with $m_{1}, n_{1}, \alpha_{1}$ and $\alpha_{2}$ set to the appropriate values listed in Ref. [10] for bromine in solids. Typically the relative charge state is greater by $5 \%$ and sometimes as high as $100 \%$ above that of a gas.

Another point of concern is that angular straggling due to scattering in helium has been disregarded. Simulations in TRIM [9] predict that $610 \mathrm{MeV}$ bromine ions coming to rest in 10 mbar of helium at $273 \mathrm{~K}$ scatter on average about $37 \mathrm{~cm}$ radially with a standard deviation of $20 \mathrm{~cm}$ over the course of their roughly $65 \mathrm{~m}$ stopping length. In the previous simulation it was demonstrated that a beam half divergence of $10 \mathrm{mrad}$ results in an extraction region with a $3 \mathrm{~cm}$ height. It seems reasonable, therefore, to expect that angular straggling would add significantly to this. The real scenario is obviously more complicated as the magnet provides a source of weak focusing which would diminish this effect somewhat. Although, this focusing becomes much weaker as the ion's charge state decreases while they lose energy. In addition, as the ions fall inward towards the end of their trajectory their motion becomes mostly radial, which is not focused vertically at all. As most angular straggling occurs towards the end of the trajectory, it is difficult to imagine that angular straggling due to ion-gas collisions would have a negligible effect.

In order to produce realistic angular straggling modeling, we have based our simulations on data from TRIM, which is itself based on an extensive experimental data set. TRIM yields, with no adjustable parameters, trajectories for arbitrary ions stopping in solids or gases. TRIM agrees well with experimental data for stopping ranges in solids and light gasses and scattering in solids, but there is very little data available for comparison with scattering in light gases. However, we have used TRIM together with the procedure presented below to reproduce experimental data for heavy ions in gas-filled magnet applications at low energies [14, 15] and find very good agreement. In particular, the effects directly related to angular straggling, which can be isolated in some applications, are satisfactorily reproduced. Additional data in this region would be extremely valuable in determining both the uncertainty in TRIM calculations and in further validating the results of this work.

TRIM allows one to produce detailed collision data files that include the position and energy of the projectile ion for each collision that results in the 


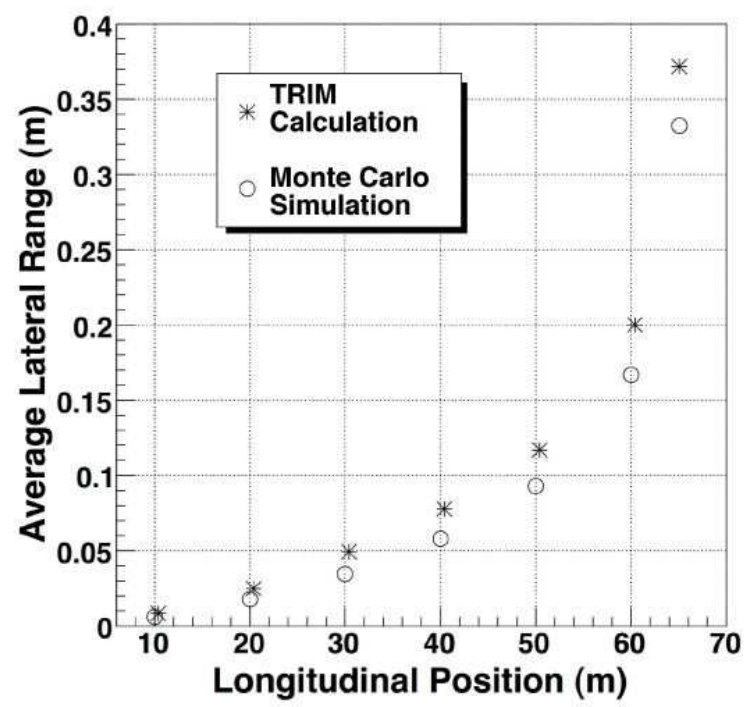

Figure 4: Plot of the average lateral range as a function of the ions longitudinal position for the Monte Carlo simulation run in SIMION and the original TRIM calculations.

displacement of a target atom. Needless to say, there are many such collisions. This collision file can be used to produce normalized velocity vectors at the vertex of each collision and by comparing the vectors at each vertex to the subsequent vector, it is possible to calculate the scattering angle at each vertex.

It is necessary to recognize, however, that the data produced by this method contains artifacts used to hasten the calculation, which convolute the data below certain energies and results in low precision data for interactions on length scales small compared to the viewing window. One can overcome this by running TRIM calculations at multiple energies, narrowing the viewing window as the energies are stepped down, and then piecing together the good high resolution data. The high resolution data can then be used to fit the collision distances and scattering angles as functions of the ion's energy.

Monte Carlo simulations using the method described above, incorporated into SIMION, produce ion trajectories that agree very well with TRIM calculations. In the absence of a magnetic field, over the $65 \mathrm{~m}$ stopping length, $610 \mathrm{MeV}$ bromine ions scattered on average about $33 \mathrm{~cm}$ transversely with a standard deviation of about $18 \mathrm{~cm}$, similar to the results of the TRIM calculations stated earlier. The results of this Monte Carlo test simulation are illustrated in Fig. 4. which shows the average lateral range as a function of the ions longitudinal position compared to calculations performed by TRIM. 


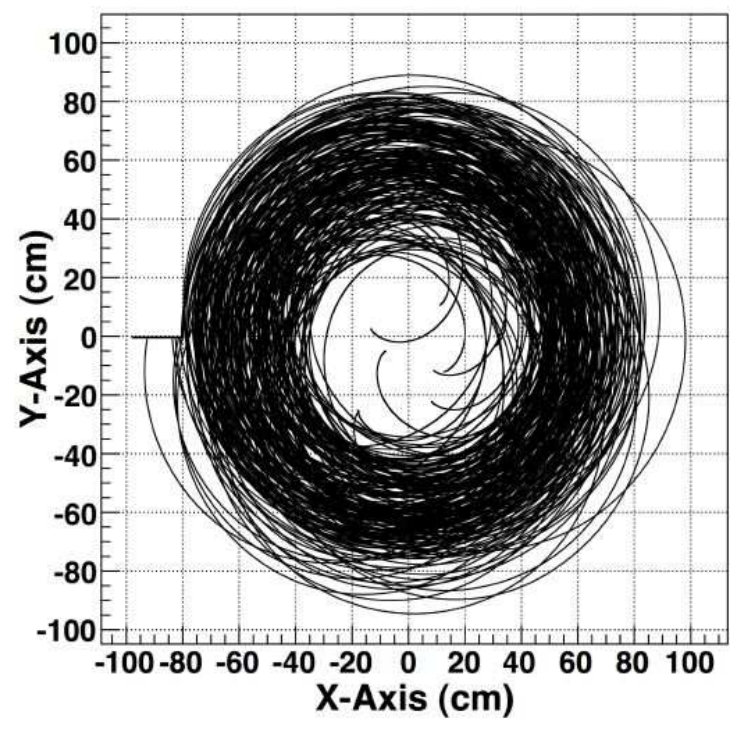

Figure 5: Trajectories of 15 ions projected onto the $\mathrm{x}-\mathrm{y}$ plane using values for $\sigma$ calculated according to Eqns. 8 \& 7

\subsection{Improved Simulations}

Let us first resolve the issue of charge-exchange cross-sections by using values for $\sigma$ calculated according to Eqns. 8 \& 7 at energies greater than $14 \mathrm{MeV}$. The results of this simulation are shown in Figs. 5, 6 \& 7 . In order to make the simulation more robust, straggling in the charge-exchange path length has been introduced according to the method discussed in the previous subsection. This addition has only a small effect on the results of the simulations.

As expected, these trajectories are much rougher than those in the previously demonstrated simulations. The spread in effective magnetic rigidities due to iongas collisions agrees very well with experimental measurements performed with the use of a gas-filled Enge spectrograph such as those reported by Paul et al. 14. In contrast, we have not been able to reconcile the previously reported "smoothed out" trajectories with either theory or experimental measurements.

In our simulation, $40 \%$ of the ions hit the degrader, significantly reducing the efficiency. In addition, the variation in trajectories due to the increased path length between charge-exchange collisions causes a slight broadening of the extraction region as well as an increase in the energy deposition within the extraction region. The ions are cooled within a disc, $35 \mathrm{~cm}$ in radius and 3 $\mathrm{cm}$ in height. Fig. 7 shows the energy deposited for a simulation where 20 of the 50 ions hit the degrader. In this simulation $407 \mathrm{MeV}$ was deposited within the extraction region. Thus, there are about 331,000 helium ions or 29 helium ions per $\mathrm{cm}^{3}$ produced within the extraction region per cooled bromine ion. The peak density of deposited energy is less than what was investigated in the 


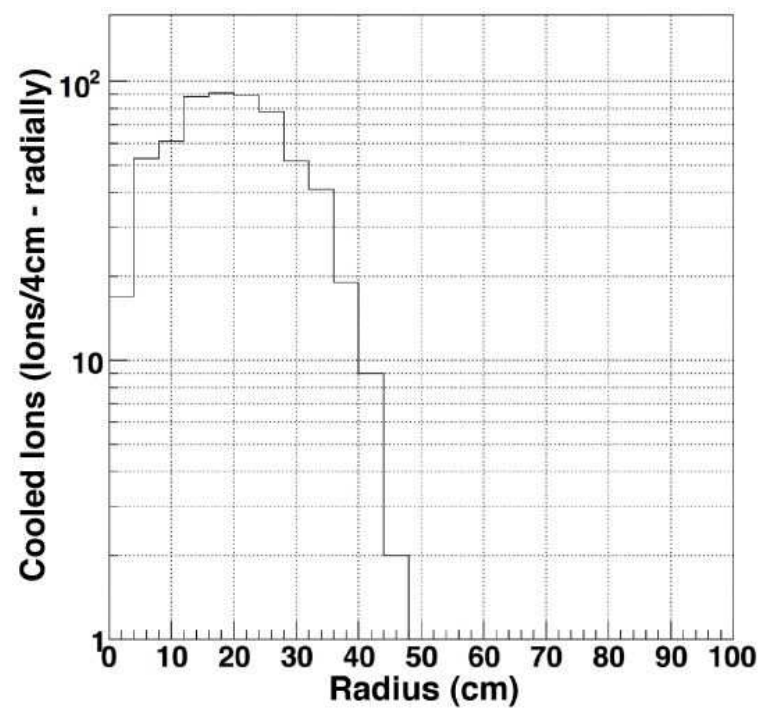

Figure 6: Histogram of the 600 cooled ions along radial direction. The density is plotted in units of ions $/(4 \mathrm{~cm}$ - radially).

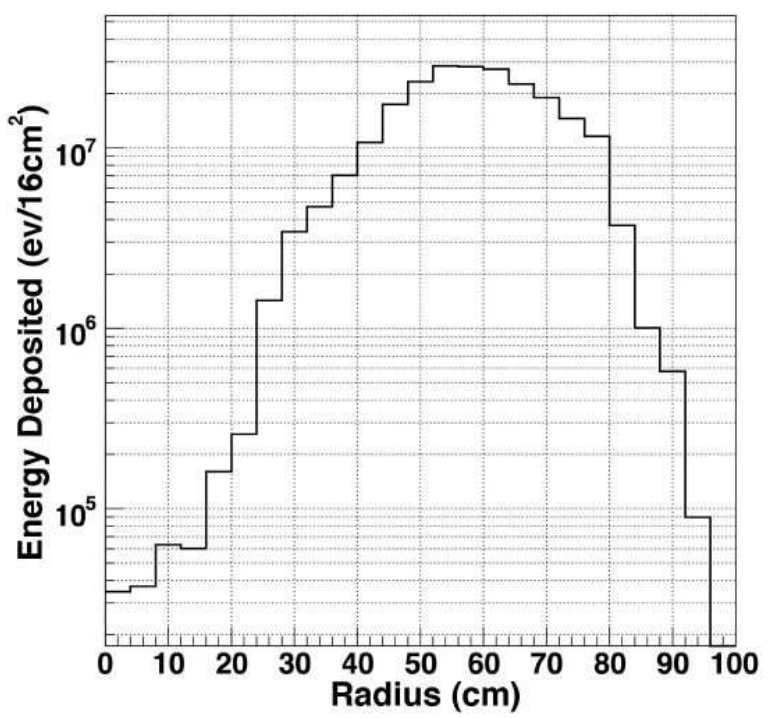

Figure 7: Total energy deposition radially along x-y plane for 50 bromine ions of which 20 hit the degrader, plotted on a logarithmic scale in units of eV/(16 $\left.\mathrm{cm}^{2}\right)$. 

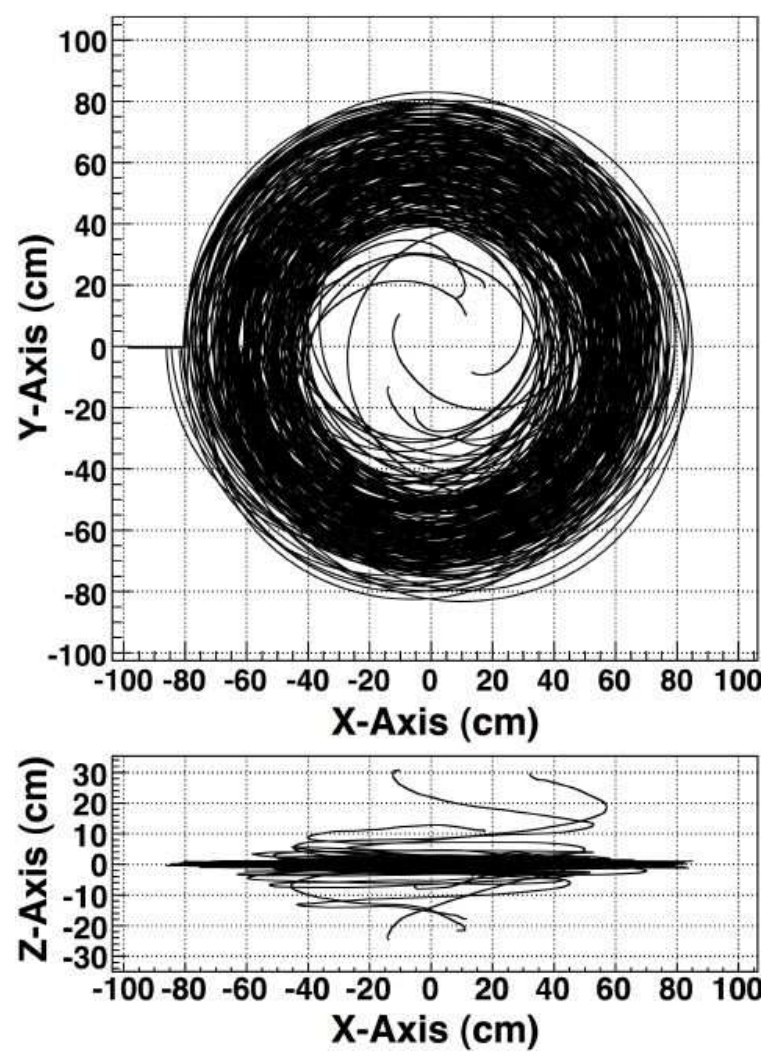

Figure 8: Trajectories of 15 ions with angular straggling included projected onto the $\mathrm{x}-\mathrm{y} \& \mathrm{x}-\mathrm{z}$ planes.

previous simulation and is therefore not of concern.

In subsequent simulations the ions were initialized with a mean charge state for bromine in solids. This was found to have little effect. In a run consisting of 1000 ions, 403 hit the degrader, a negligible change from the previous simulation. This is not too surprising when one considers that the cross-section for chargeexchange collisions is dominated by an exponential as ions drifts away from the mean charge state.

The most drastic change to the simulation comes from the addition of angular straggling, introduced using the method described in the previous subsection. Unlike the previous simulations, it is important to note that the pole gap of the magnet used in the simulation was increased to $70 \mathrm{~cm}$ as most of the ions were hitting the top and bottom of the magnet. Fig. 8 shows the trajectories of 15 ions projected onto the $\mathrm{x}-\mathrm{y}$ and $\mathrm{x}-\mathrm{z}$ planes of which 6 ions hit the degrader. Fig. 9 illustrates a density plot of the cooled ions for a run consisting of 1000 ions, in which 416 hit the degrader or the walls of the magnet.

In these simulations the magnetic field was calculated using Eqns. 1$]$ \& 2. 


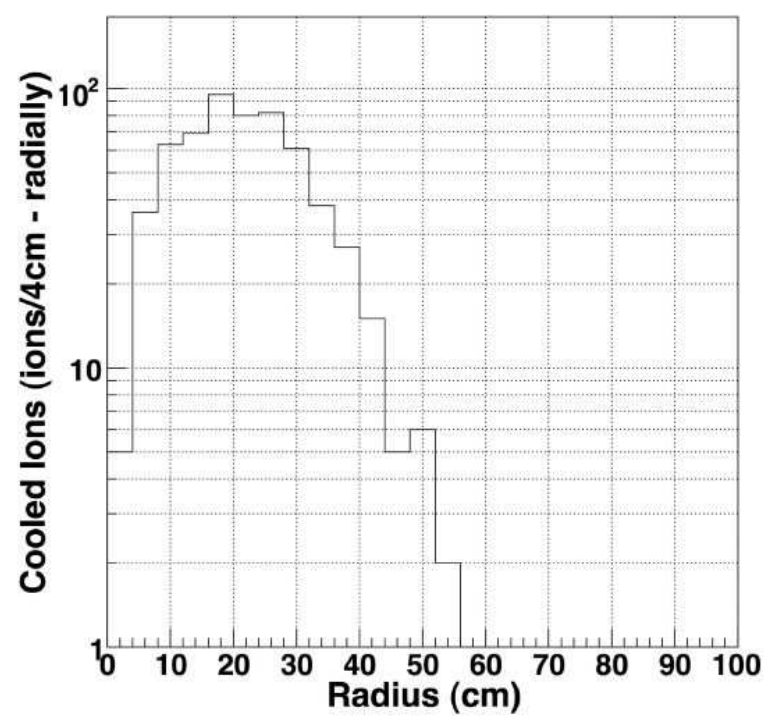

Figure 9: Histogram of the 584 cooled ions along the radial direction plotted in units of ions $/\left(16 \mathrm{~cm}^{2}\right)$.

which were derived in the paraxial approximation. It is not clear that these expressions are still valid for simulations of such large gap magnets. In order to verify their validity, the magnetic fields of three large gap magnets of the same specifications and $1 \mathrm{~m}$ radii, but varying pole gaps, were modeled in SIMION. Fig. 10 shows the percentage of cooled ions as a function of the magnet pole gap for simulations using both the analytical expressions of Eqns. 1 \& 2 and the magnetic fields produced by SIMION. There are only a few data points for simulations using the SIMION modeled magnetic fields since these simulations require a new magnet geometry to be compiled for each specific pole gap. Fig. 10 demonstrates that the analytical expressions used to calculate the magnetic fields are sufficient even for the largest pole gaps used in these simulations.

From Fig. 10, it is clear that a stopping efficiency below $1 \%$ is obtained with the previously proposed $4 \mathrm{~cm}$ electrode gap. The maximum achievable stopping efficiency of $60 \%$ is limited mainly by losses in the degrader. This comes before taking into account further losses due to neutralization and extraction. Achieving efficiencies in this range requires the construction of a 2 Tesla cyclotron magnet with a $60 \mathrm{~cm}$ pole gap. In practice, the actual gap would have to be of the order of $70 \mathrm{~cm}$ in order to house the necessary equipment that such a concept would require. This poses many difficult engineering challenges as well as result in a significant increase in cost.

Fig. 11] shows the energy deposited from a run in which 27 out of 50 ions were successfully cooled. The maximum density of deposited energy per cooled ion is found to be about $70 \mathrm{keV} / \mathrm{cm}^{2}$, a reduction by a factor of about 1.8 compared to the simulation illustrated in Fig. 7. In the previous simulations 


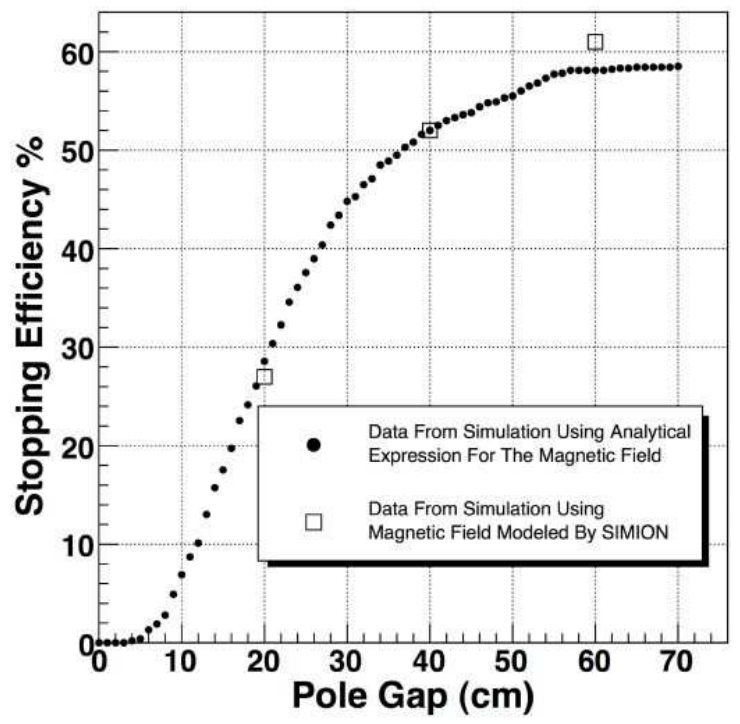

Figure 10: Stopping efficiency as a function of magnet pole gap.

this charge was confined within the $4 \mathrm{~cm}$ gap, allowing for quick extraction of bulk Helium ions. A new analysis is required, however, for this new magnet geometry. According to 8 the He ion space-charge, while moving towards the charge collection electrodes, induces a voltage:

$$
V_{\text {ind }}=\sqrt{\frac{e Q}{4 \varepsilon_{0} k}} d^{2}
$$

Where, $e$ is the electric charge, $Q$ is the rate of ionization per volume, $\varepsilon_{0}$ is the permittivity of free space, $d$ is the distance between charge collection electrodes, and $k=k_{0}(\mathrm{He}) \cdot(1013 \mathrm{mbar}) / P_{\mathrm{He}}$ is the mobility of helium ions at a given pressure $P_{\mathrm{He}}\left(k_{0}(\mathrm{He})=1.05 \cdot 10^{-3} \mathrm{~m}^{2} /(\mathrm{Vs})\right)$.

For a $60 \mathrm{~cm}$ gap with helium pressure of $10 \mathrm{mbar}$, the Paschen curve in helium indicates a breakdown voltage of roughly 1800 volts, probably lower in the presence of magnetic fields. This corresponds to an electric field of about $30 \mathrm{~V} / \mathrm{cm}$ across the gap, the same electric field used in the previous rate calculations. Thus, we find that while the reduction in the peak density of deposited energy will increase the beam intensity tolerance by a factor of roughly 1.8 , the 15 times larger gap will reduce the ion intensity this device can tolerate by about a factor of 15. An overall reduction of about a factor of 10 is expected within the formalism of Eqn. 11. However, with the gap size being now larger than the radial distance between the stopping volume and the bulk of the ionization, it is to be expected that some of the ionization will be repelled into the extraction volume. While this can easily be handled if the ionization remains in the form of helium ions since those are not transported by the RF carpet, even a small fraction of this ionization creating contaminant ions by charge exchange processes 


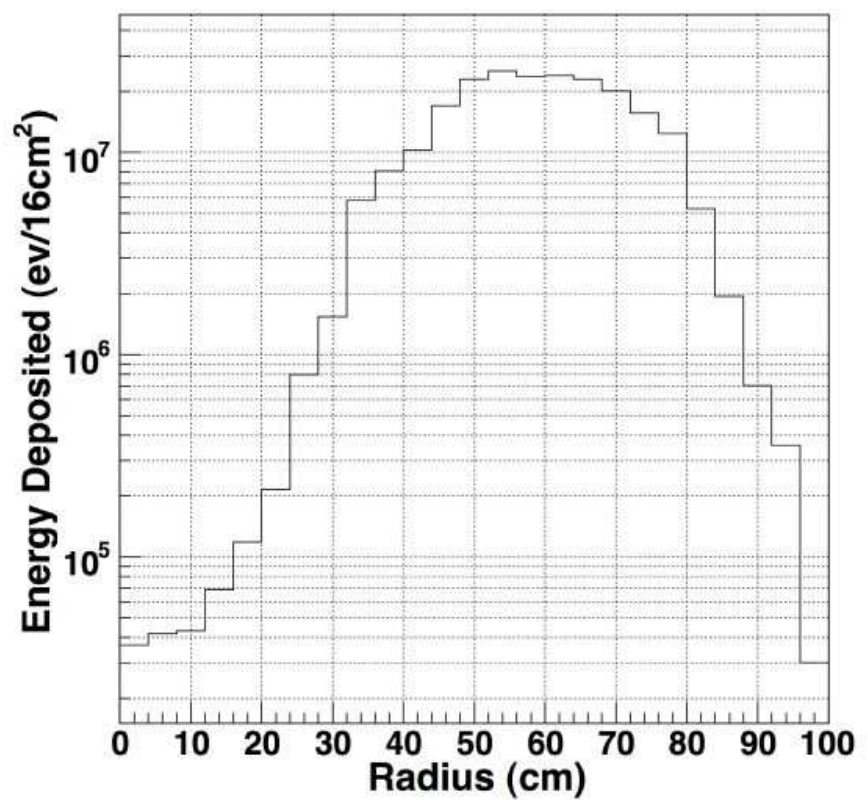

Figure 11: Total energy deposition along radial direction for 50 bromine ions of which only 27 were successfully cooled. The density is plotted on a logarithmic scale in units of $\mathrm{eV} /\left(16 \mathrm{~cm}^{2}\right)$. 
could easily saturate the extraction region. A more complete description and accurate calculation of this effect is presented later in the text for a proposed improved geometry with more details given in [16] in preparation.

Neglecting space-charge effects, extraction times for $\mathrm{Br}$ ions become only slightly longer across the $70 \mathrm{~cm}$ gap. For a $30 \mathrm{~V} / \mathrm{cm}$ electric field the ion drift velocity is about $600 \mathrm{~m} / \mathrm{s}$. Using this result together with the times for extraction along the RF carpet, funnel, and linear RFQ guide to a point outside the magnet as reported in Ref. [8], one finds that it would take roughly 5-10 ms to extract ions across the $70 \mathrm{~cm}$ pole gap, comparable to the times stated in Ref. [8].

These calculations do not take into account effects on extraction times that arise from the build up of space charge within the extraction region. Within the extraction region, a disc $40 \mathrm{~cm}$ in radius and $70 \mathrm{~cm}$ in height, a total of $1.25 \mathrm{GeV}$ was deposited for the run in which 27 out of 50 ions were successfully cooled, corresponding to about $1.1 \cdot 10^{6}$ helium ions per cooled bromine ion or about 3 helium ions per $\mathrm{cm}^{3}$. This charge as well as charge flowing into the extraction region from the region of bulk ionization will certainly result in a reduction of extraction efficiencies, a reduction in allowed beam intensities, and an increase in extraction times.

All of the simulations discussed thus far have been performed using a small beam-spot with an average energy spread $\Delta \mathrm{E} / \mathrm{E}$ of $20 \%$ at $8 \mathrm{MeV} / \mathrm{u}$, a beam half width of $5 \mathrm{~mm}$ and a beam half divergence of $10 \mathrm{mrad}$ after passing through the degrader foil. These parameters account for only a small fraction of the desired reaction products which are produced with a large variance in both beam energy and angular divergence. The fragment separator in use at current medium energy fragmentation facilities have momentum acceptance of roughly $5 \%$ with angular acceptances of $\pm 50 \mathrm{mrad}$ in both the $\mathrm{x}$ and $\mathrm{y}$ directions for a $1 \mathrm{~mm}$ spot. Various techniques for momentum compression have been discussed at great length in the literature [17, 18, 19] and designs for proposed new facilities [20] extend this momentum acceptance to $10 \%$ with similar angular acceptance. An initial beam energy of $150-200 \mathrm{MeV} / \mathrm{u}$ is required to yield the $100 \mathrm{MeV} / \mathrm{u}$ mass separated recoils at the end of the fragment separator. The selection process through the fragment separator will have the recoils going through degraders of roughly half the range of the selected fragment so that according to 21] the beam-spot size at the end of the separator is increased by a factor of 2 over that without degraders. The initial $1 \mathrm{~mm}$ beam-spot with \pm $50 \mathrm{mrad}$ angular envelope and no energy spread therefore fills already at this intermediate energy the full $1 \mathrm{~cm}$ beam-spot with $\pm 10 \mathrm{mrad}$ assumed in [8] and used so far in this paper. Adding the effect of the $5 \%$ momentum spread acceptance of current fragment separators, a momentum dispersive system and a wedged monochromatic degrader are required to minimize the energy spread at the degrader. The small angular acceptance used in the simulations limits the resolution that can be achieved in this last stage so that a momentum dispersion of at least $2 \mathrm{~cm} / \%$ is required leading to a beam-spot size of at least $10 \mathrm{~cm}$ high (dispersion is along the vertical axis) by $1 \mathrm{~cm}$ wide on the degrader, even for current separators. 


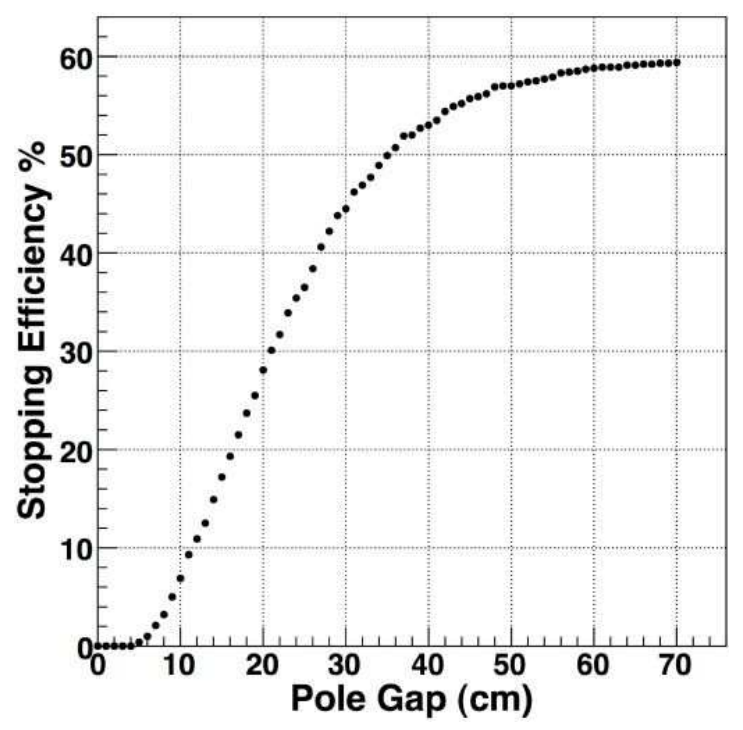

Figure 12: Stopping efficiency as a function of pole gap for simulation using a $1 \mathrm{~cm}$ by $10 \mathrm{~cm}$ beam-spot.

Fig. 12 shows the percentage of cooled ions as a function of the magnet pole gap for the simulation performed with the $1 \mathrm{~cm}$ by $10 \mathrm{~cm}$ beam. It could have been chosen to widen the beam along the $\mathrm{x}$-axis rather than the $\mathrm{z}$-axis, however, this would result in very limited gains as most of the added beam would return and hit the degrader foil.

Contrary to what one would naively expect, Fig. 12 shows a slight increase in the maximum stopping efficiency as compared to Fig. 10. There are several factors that contribute to this. First, the relative phase of oscillations along the $\mathrm{z}$-axis and $\mathrm{x}$-axis are aligned such that the maximum velocity along the $\mathrm{z}$ direction, and therefore the minimum in the $x-y$ plane, is achieved after slightly more than half a rotation in the $\mathrm{x}-\mathrm{y}$ plane. Thus, as ions finish their first rotation they do so with a slightly reduced cyclotron radius. This reduces the likelihood of ions hitting the degrader, particularly for ions that start further off-center. Furthermore, the limitations in the efficiency of the system are dominated by the loss of ions due to large angular straggling as well as losses due to ions hitting the degrader after one or more rotations. The restoring force of the magnet along the $\mathrm{z}$-direction is such that ions starting at $z=0$ with $10 \mathrm{mrad}$ divergence relative to the $\mathrm{x}-\mathrm{y}$ plane will oscillate back and forth from $\pm 1.5 \mathrm{~cm}$. When the ions start more than $2 \mathrm{~cm}$ off-center along the $\mathrm{z}$-axis the ions will oscillate with an amplitude approximately equal to their starting position. It is only in the final several meters of the ions flight that appreciable scattering takes place resulting in the shape of the curves seen in Figs. 10] \& 12.

A thorough analysis of the relative phase of oscillations along the $\mathrm{z}$ and $\mathrm{x}$ directions reveals a method for limiting losses in the degrader. Fig. 13 illustrates 
initial oscillations along both axis for an ion starting at $\mathrm{z}=3 \mathrm{~cm}$. From this plot it is evident that the phases of the two oscillations are aligned such that an ion starting at $\mathrm{z}=3 \mathrm{~cm}$ reaches $\mathrm{z}=-3 \mathrm{~cm}$ very close to where the ion finishes its first rotation in the $\mathrm{x}-\mathrm{y}$ plane. That is to say, oscillations along the $\mathrm{z}$-axis occur at about twice the cyclotron period. In this type of configuration the ion illustrated in Fig. 13 would hit the degrader as it returned on its first rotation. However, if one moves the incoming beam and the degrader so they are located at $\mathrm{z} \geq 0$, the same ion misses the degrader on its first pass and the combination of cooling and a slight procession in the orbit causes it to just miss the degrader on the second pass. At injection the cyclotron frequency and the frequency of oscillations in the z-direction (in the paraxial and small angle approximations) are given by:

$$
\begin{gathered}
\omega_{c} \approx \frac{q B_{o}\left(1-n_{o}\right)}{m} \\
\omega_{z} \approx \sqrt{\frac{q v B_{o} n_{o}}{m r_{\mathrm{inj}}}}
\end{gathered}
$$

The ratio of the frequencies is given by:

$$
\frac{\omega_{z}}{\omega_{c}} \approx \sqrt{\frac{m v n_{o}}{q B_{o} r_{\mathrm{inj}}}}\left(1-n_{o}\right)^{-1}=\sqrt{\frac{r_{c} n_{o}}{r_{\mathrm{inj}}\left(1-n_{o}\right)}}
$$

Where $v$ is the initial ion velocity, $m$ is the ion's mass, $q$ is the charge of the ion, $n_{o}$ is the field index (as defined in Eqns. 11 \& 2), $B_{o}$ is the peak magnetic field, $r_{\text {inj }}$ is the injection radius, and $r_{c}$ is the characteristic cyclotron radius of the ion upon injection. Thus, the ratio of the vertical focusing frequency versus the cyclotron frequency for the initial orbits is a constant for this magnetic field configuration for all particles whose initial cyclotron radius corresponds to the .8 $\mathrm{m}$ injection radius used in this configuration. This approach of offsetting in the vertical direction the injection point and degrader is therefor generally applicable to all ion species by setting the injection energy, or scaling the magnetic field, to obtain the appropriate initial cyclotron radius.

Fig. 14 illustrates the stopping efficiency for two simulations. The first simulation has a $1 \mathrm{~cm}$ beam-spot offset by $2 \mathrm{~cm}$ along the $\mathrm{z}$-axis, with the degrader located at $\mathrm{z} \geq 1.5 \mathrm{~cm}$. The second simulation has a beam-spot $1 \mathrm{~cm}$ wide, $10 \mathrm{~cm}$ tall, and is offset by $5.5 \mathrm{~cm}$ along the z-axis, with the degrader located at $\mathrm{z} \geq 0.5 \mathrm{~cm}$. These types of configurations significantly reduce losses in the degrader and greatly improve the overall stopping efficiencies (but the large magnet gap is still required). A maximum achievable stopping efficiency approaching $90 \%$ and $80 \%$ has been observed for the $2 \mathrm{~cm}$ offset beam and the much larger acceptance offset $1 \mathrm{~cm}$ by $10 \mathrm{~cm}$ beam, respectively, for a $70 \mathrm{~cm}$ gap. The cost and design complications of such a magnet would be high and require one to consider the tradeoff between efficiency and the size of the pole gap. A more modest $30 \mathrm{~cm}$ gap would allow for a stopping efficiency of about $65 \%$ and $60 \%$ for the $2 \mathrm{~cm}$ offset beam and the offset $1 \mathrm{~cm}$ by $10 \mathrm{~cm}$ beam respectively. 


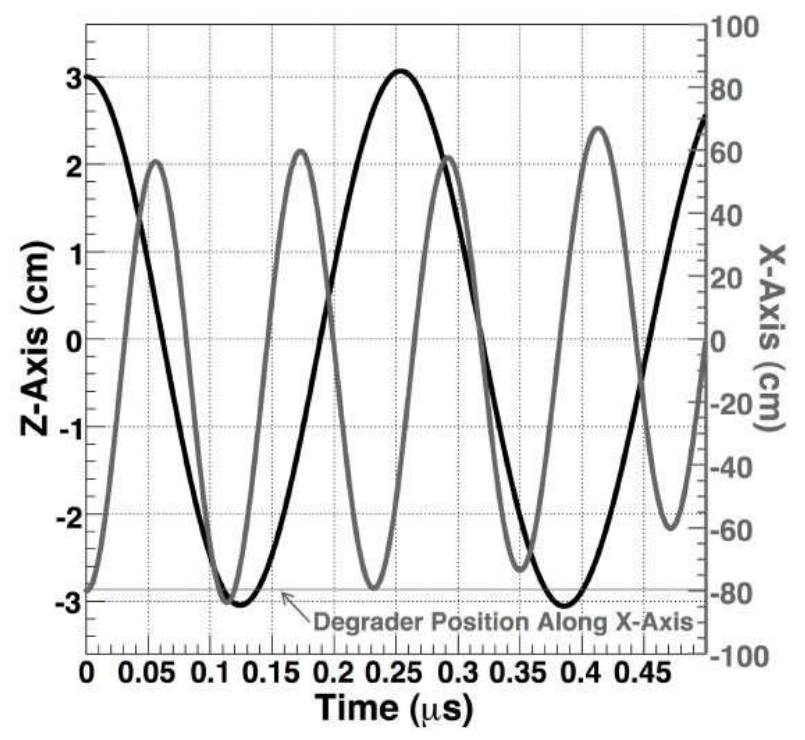

Figure 13: Position along z-axis(black) and x-axis(grey) as a function of time. The grey line at the bottom represents the degrader located at $\mathrm{x} \leq-79.5 \mathrm{~cm}$.

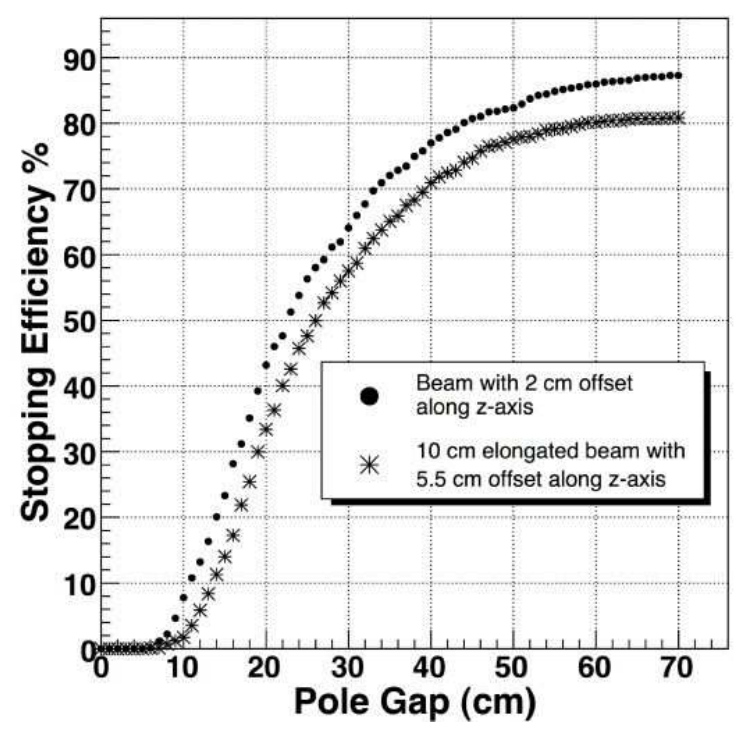

Figure 14: Stopping efficiency as a function of pole gap using offset beams. 
A detailed analysis of the space charge effect has been performed for a 60 $\mathrm{cm}$ pole gap with an offset large acceptance $1 \mathrm{~cm}$ by $10 \mathrm{~cm}$ beam. The energy deposited by the decelerating ions is converted to a charge creation rate per unit volume using the known average energy required per ion-electron pair creation of $42 \mathrm{eV}$ in helium gas. The electrons move roughly 1000 times faster than the helium ions in the gas under the influence of an electric field and are neglected since they only contribute insignificantly to any space charge buildup. The trajectories of the He ions created by the energy loss are calculated in the electric field created by the electrodes (and the magnetic field) and a "static" space charge distribution is built from the time averaged space-charge density they create. The electric fields created by this space-charge distribution is then calculated. The procedure is then repeated with further ion creation and transport now in the combined electrode-generated and space-charge-generated electric fields and the resulting space-charge density is recalculated. The procedure is repeated until the problem converges to a self-consistent solution (more details will be available in [16]).

The main results of these calculations for the geometry of interest are shown in Figs. 15 \& 16, which depict the applied DC potentials modified by spacecharge buildup at different radii for incoming ion beam intensities of $1 \cdot 10^{8}$ and $5 \cdot 10^{8}$ ions per second respectively. At $1 \cdot 10^{8}$ ions per second, the fields are modified but essentially behave like the case with no space charge where the plot would show a straight line from 1800 to 0 volts going across the plot. All ions are pushed towards the extraction region and fields are large enough to avoid neutralization and keep extraction times short enough to limit charge exchange with contaminants.

In the extraction region the ions are transported along a $40 \mathrm{~cm}$ radius $\mathrm{RF}-$ carpet towards an extraction RFQ. The DC-field perpendicular to the RF-carpet is on the order of $40 \mathrm{~V} / \mathrm{cm}$, similar to the case where no space-charge is present. While no RF-carpet has operated under these exact conditions, the general scaling properties of the RF-force are well known [2] and for the correct pressure and frequency regime the force goes as one over the pressure squared and is proportional to the ion mass. Operation with gradients in excess of $20 \mathrm{~V} / \mathrm{cm}$ for masses in the range of roughly $100 \mathrm{AMU}$ has been demonstrated at pressures in excess of 100 mbar with an RF-cone operating at $2 \mathrm{MHz} 22$, as has operation at fields of up to $18 \mathrm{~V} / \mathrm{cm}$ at a pressure of about $100 \mathrm{mbar}$ for ions of mass 8 with an RF-carpet operating at $15 \mathrm{MHz}$ [2]. Both suggest, when taking into account the much lower 10 mbar operating pressure required here, the ability to handle fields well in excess of $50 \mathrm{~V} / \mathrm{cm}$ for the mid mass nuclei considered here. It is expected from these results that the device can operate without severe disturbance at $1 \cdot 10^{8}$ incoming ions per second with little effect on extraction time and efficiency.

At $5 \cdot 10^{8}$ ions/s, the potential from the space charge is severe enough to have created a potential maximum with the ions in the lower half of the device (the negative z-axis on the plot) now pushed down, away from the extraction $\mathrm{RFQ}$, instead of up. In the upper half (the positive z-axis of the plot) where the ions are still directed towards the extraction RFQ, the electric gradient 


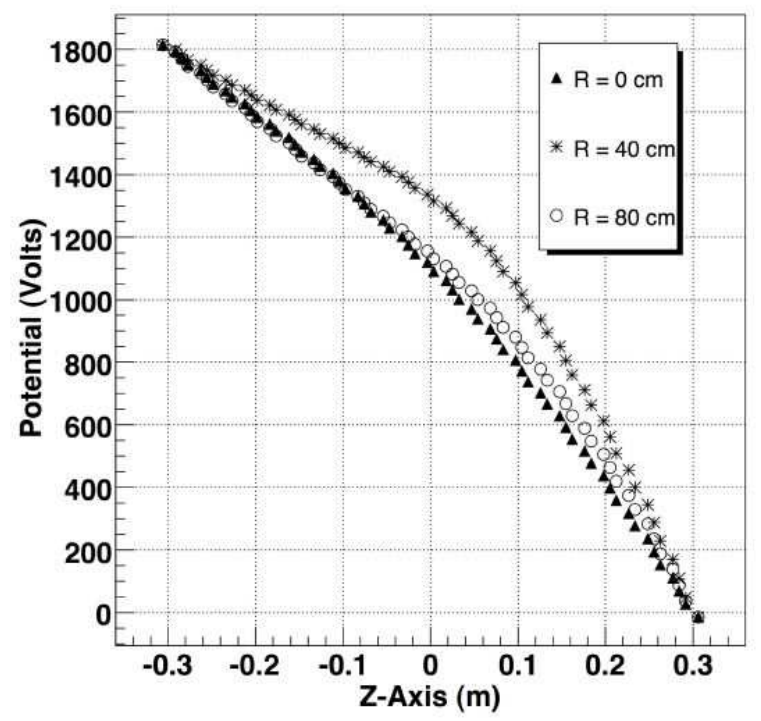

Figure 15: Total electrostatic potential along z-axis coming from both the applied collection potential and the Induced space charge potential at various radial distances for $1 \cdot 10^{8}$ incident $\mathrm{Br}$ ions per second.

is now much larger, which may lead to a decrease in the Br extraction time. The RF-focusing structure on the pole may still be strong enough to repel the heavy ions because of the low operating gas pressure (the He ions are not repelled). More importantly, helium ions created in the central region where the electric potential has a maximum spend a large period of time, up to hundreds of millisecond, in the gas before being extracted and are much more likely to charge exchange with contaminants in the gas which will then be carried out to the extraction RFQ and lead to saturation. Radioactive ions stopped in the resulting low electric field central region are also more likely to neutralize or undergo charge-exchange reactions leading to further losses. All of these effects are still negligible at $1 \cdot 10^{8}$ incoming ions per second which we determine as the practical operation limit for this geometry. This device has a larger stopping range than any demonstrated gas catchers and a space-charge limit that far exceeds that demonstrated for all other large gas catcher except for the recent high-intensity gas catcher developed at ANL [23]. The large stopping range make the proposed cyclotron stopper especially well suited to the lightest species who have low stopping power and are therefore difficult to stop in a linear gas catcher.

Alternative methods of focusing have also been investigated in an attempt to minimize the effects of angular straggling. Simulations using magnets with increased field indexes failed to produce significant increases in efficiencies. However, split sector magnets were found to have several benefits. 


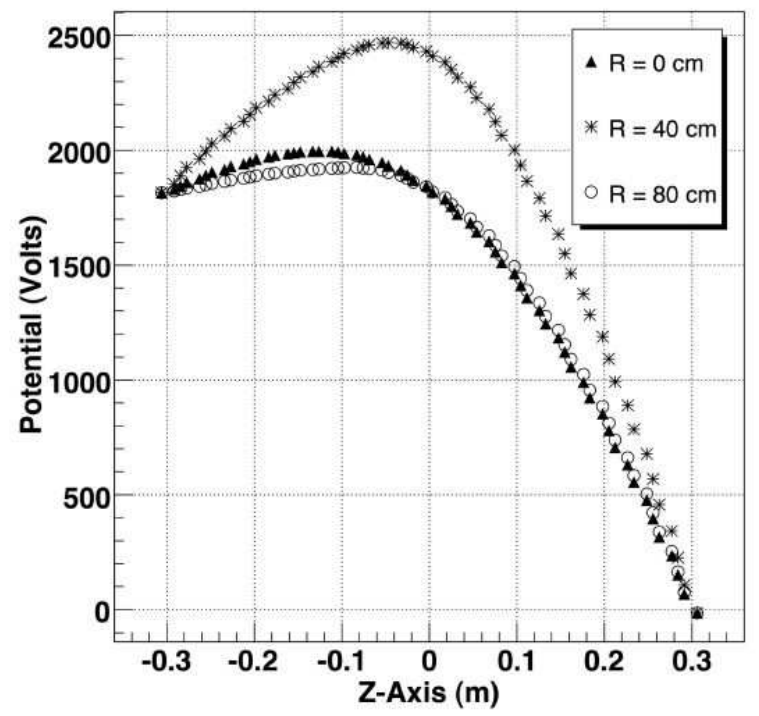

Figure 16: Total electrostatic potential along z-axis coming from both the applied collection potential and the Induced space charge potential at various radial distances for $5 \cdot 10^{8}$ incident $\mathrm{Br}$ ions per second.

Split sector magnets and their fields were modeled in SIMION. Fig [17]shows a mapping of $B_{z}$ along the primary plane of a $1.5 \mathrm{~m}$ radius split sector magnet with a $30 \mathrm{~cm}$ pole gap, $20 \mathrm{~cm}$ wide pole magnets, and a nominal field strength of 2 Tesla. The split sector magnet simulations required a few adjustments from previous simulations, namely, the optimal radius of injection was found to be between $90 \& 110 \mathrm{~cm}$ depending on the gap of the magnet.

Fig. 18]shows the trajectories of 15 ions from a $1 \mathrm{~cm}$ centered beam projected into the $\mathrm{x}-\mathrm{y}$ plane and $\mathrm{x}-\mathrm{z}$ plane for the magnet whose field is illustrated in Fig. 17. Notice that one of the ions fell out of the stable orbit. The orbits of the split sector design are much more sensitive to variations in the ions initial conditions as well as their effective magnetic rigidities, the profile of which is determined by the details of the charge exchange collisions. In a simulation with 1000 ions, this effect contributed to about $2 \%$ of losses. The rest of the losses were due to angular straggling or losses in the degrader. Losses in the degrader, however, were significantly reduced as compared to the previously investigated magnet design. Fig. 19] shows the percentage of cooled ions as a function of the magnet gap width for three different configurations; One with a $1 \mathrm{~cm}$ beam on center, one with a $1 \mathrm{~cm}$ beam-spot offset by $2 \mathrm{~cm}$, and another with a $1 \mathrm{~cm}$ by $10 \mathrm{~cm}$ beam-spot offset by $5.5 \mathrm{~cm}$ along the z-axis. Unlike previous simulations where analytical expressions for the magnetic field were used, different pole gaps result in different field profiles due to the lack of rotational symmetry about the z-axis. It was therefore necessary to run a separate simulation for each pole gap in order 


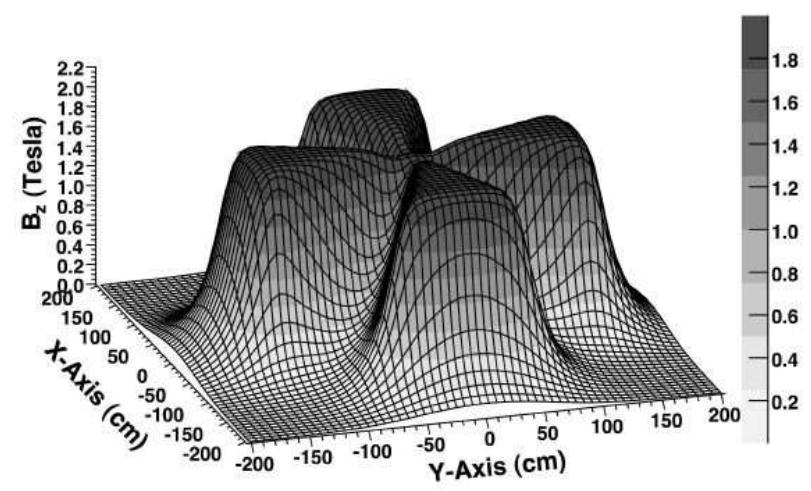

Figure 17: Map of $B_{z}$ for a split sector magnet with a $30 \mathrm{~cm}$ gap and $20 \mathrm{~cm}$ poles. The field strength is plotted on a linear scale and reaches a maximum value of 2 Tesla.

to take into account the varying field profiles. As a result, Fig. 19 has a limited number of data points compared to Figs. 10, 12 \& 14,

For centered beams the split sector design increases overall efficiencies by reducing the loss of ions due to collisions with the degrader, but the maximum achievable efficiency when the beams are offset is about $85 \%$ for a $60 \mathrm{~cm}$ pole gap, roughly the same as the previously studied magnets. The apparatus does, however, demonstrate more effective focusing, which is evident by the gains in efficiency of more than $35 \%$ for smaller pole gaps. The main drawback to this method is the large extraction region and the increase in energy deposited within this region. In this design ions move in elliptical orbits whose centers tend to drift away from the center of the magnet. In some cases the ions form highly eccentric orbits causing them to come very near the center of the magnet and depositing large amounts of energy within the extraction region during half of their rotation. While losing energy the orbits shrink about their center and in extreme cases some ions fail to make it back around the center of the magnet causing them to fall out of the system as seen in Fig. 18 Fig. 20 shows the energy deposited from a run with a $5 \mathrm{~mm}$ half width centered beam using the magnet whose field is illustrated in Fig. 17. In this run 28 out of 50 ions were successfully cooled. The maximum density of deposited energy per cooled ion is found to be about $45 \mathrm{keV} / \mathrm{cm}^{2}$, a nearly 3 fold reduction compared to the simulation illustrated in Fig. (7) For the $30 \mathrm{~cm}$ gap used in this simulation, the resulting beam intensity limit is reduced by a factor of 2 to 3 from what was predicted in 8 . This, however, does not take into account that nearly half of the energy is deposited within the extraction region, where $7.5 \mathrm{GeV}$ was deposited. This corresponds to $6.5 \cdot 10^{6}$ ionized helium atoms per cooled bromine ion. There is no doubt that this will place severe limits on extraction efficiencies and times as well as allowed beam intensities. 

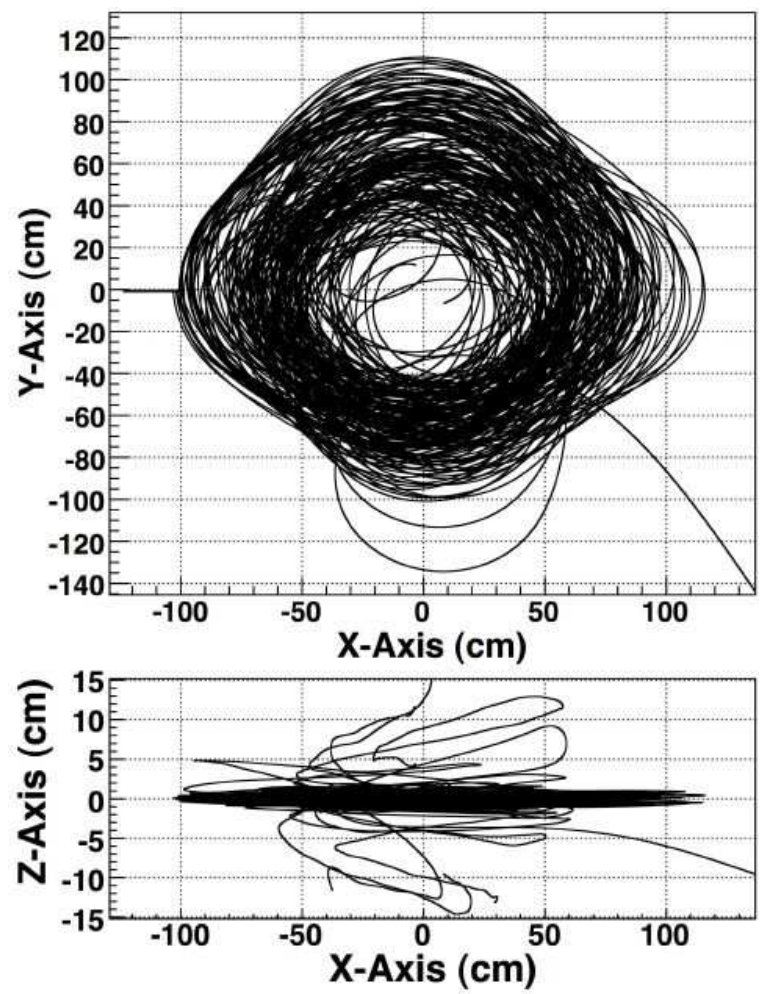

Figure 18: Trajectories of 15 ions projected onto the x-y \& x-z planes using split sector magnet with $30 \mathrm{~cm}$ pole gap. 


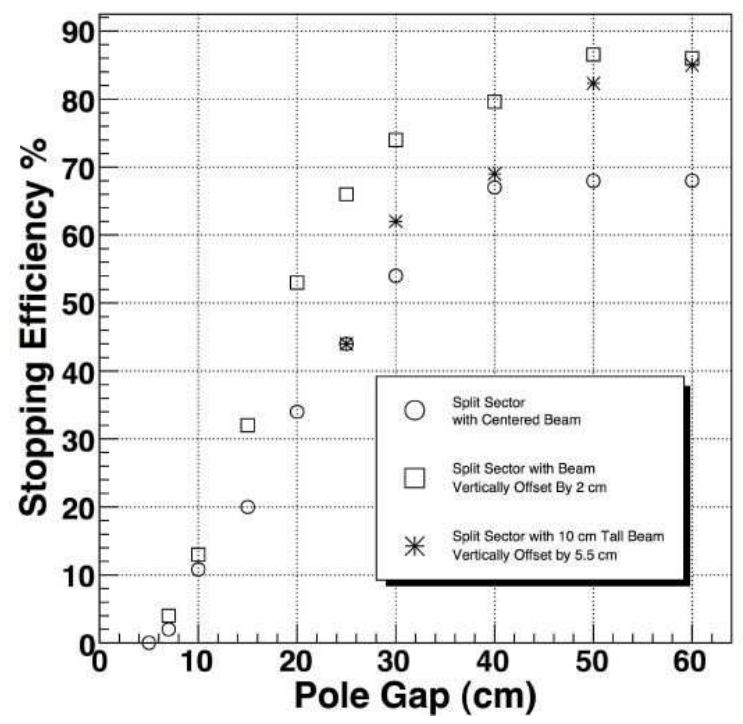

Figure 19: Stopping efficiency as a function of pole gap.

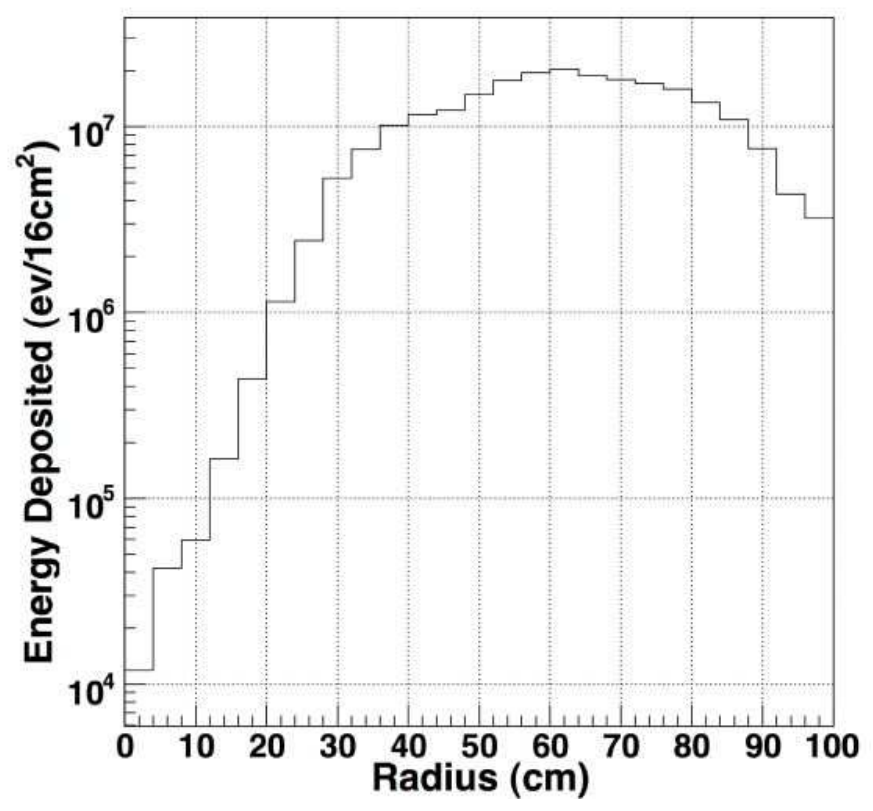

Figure 20: Total energy deposition along radial direction for 50 bromine ions of which only 28 were successfully cooled, plotted on a logarithmic scale in units of $\mathrm{eV} /\left(16 \mathrm{~cm}^{2}\right)$. 


\section{Conclusion}

Extensive simulations of the cyclotron stopper have been performed including physics processes that were either omitted or incorrectly treated in Ref. [8]. While it is difficult to place uncertainties on these calculations due to the small amount of experimental data available for the scattering of heavy ions in light gases, the results of these simulations agree well with the limited experimental data on gas filled spectrometers and charge-exchange cross-sections. These results are at variance with the simulations reported in Ref. [8]. We find the efficiency for the geometry proposed in that paper to be lowered by more than two orders of magnitude by the better treatment of the ion-gas interactions.

Increasing the pole gap of the magnet by a factor of about 15 allows one to recover a stopping efficiency of roughly $60 \%$. In addition, the factor of 15 increase in pole-gap required to handle the angular straggling allows the use of an offset degrader, which provides a further increase in the maximum achievable stopping efficiency approaching 90\%. Precise experimental determination of the radial straggling at low energies would be usefull to further refine these simulations. The newly proposed geometry results in a similar extraction time and a similar order of magnitude maximum intensity limit for the operation of this device. The final extraction efficiency, however, will likely be lower.

This paper, to maintain a direct comparison to Ref. [8], only quotes stopping efficiencies. Not mentioned in both is the loss of ions due to neutralization in the stopping process. For stopping in He, several experiments suggest a maximum achievable neutralization survival probability [24, 25] during the process of slowing down in the range of $30-50 \%$ depending on the ion being cooled. Achieving efficiencies in this range is not a trivial task as it requires sub-ppb impurity levels within the stopping volume. Such efficiencies have only been accomplished by cryogenically freezing out any possible contaminants that may neutralize the ions (temperatures below $100 \mathrm{~K}$ were necessary to fully minimize the effects of neutralization in $\mathrm{He}$ ) [24] or through the use of ultra high purity helium in an ultra high vacuum system [25. In addition, there might also be losses due to inefficiencies in extraction that will arise at high intensity operation from the increased space-charge effects due to the large pole gap. For operation below the space charge limit, the overall efficiencies should be comparable to linear gas catchers, but with a much longer stopping range that is particularly useful for light ions. The main technical challenge with this approach will be the construction of such a large magnet geometry.

Finally, it has been found that the limited acceptance of the previously proposed apparatus can be increased to cover a large fraction of the acceptance of modern fragment separator with very little additional loss in efficiency by using an offset degrader for this wider gap design. The space-charge handling capabilities for such a geometry are calculated to be about $10^{8}$ incoming ions per second, more than adequate for most applications. 


\section{Acknowledgments}

This work was supported by the U.S. Department of Energy, Nuclear Physics Division, under contract No. DE-AC02-06CH11357.

\section{References}

[1] G. Savard, et al., Nucl. Inst. and Meth. B 204 (2003) 582.

[2] M. Wada et al., Nucl. Inst. and Meth. B 204 (2003) 570.

[3] G. Sikler, et al., Nucl. Inst. and Meth. B 204 (2003) 482.

[4] L. Weissman, et al., Nucl. Inst. and Meth. A 540 (2005) 245.

[5] M. Huyse et al., Nucl. Inst. and Meth. B 187 (2002) 535.

[6] A. Takamine et al., Rev. Sci. Inst. 76, 103503 (2005).

[7] I. Katayama et al., Hyperfine Interactions 115 (1998) 165.

[8] G. Bollen et al., Nucl. Inst. and Meth. A 550 (2005) 27.

[9] J.F. Ziegler, SRIM-2003, http://www.SRIM.org/SRIM

[10] H.D. Betz, Rev. Mod. Phys. 44 (1972) 465.

[11] Scientific Instrument Services, Inc., SIMION 7, http://www.SIMION.com

[12] J. Alonso et al., Transactions on Nucl. Sci. Vol. NS-26, No. 3 (1979) 3686.

[13] W.G. Graham et al., J. Phys. B: At. Mol. Phys. 18 (1985) 2503.

[14] M. Paul et al., Nucl. Inst. and Meth. A 277 (1989) 418.

[15] K.E. Rehm et al., Nucl. Inst. and Meth. A 344 (1994) 614.

[16] M. Sternberg et al., to be published.

[17] H. Geissel et al., Nucl. Inst. and Meth. A 282 (1989) 247.

[18] H. Weick et al., Nucl. Inst. and Meth. B 164 (2000) 168.

[19] C. Scheidenberger, et al., Nucl. Inst. and Meth. B 204 (2003) 119.

[20] B.M. Sherrill, Nucl. Inst. and Meth. B 204 (2003) 765.

[21] J.P. Dufour et al., Nucl. Inst. and Meth. A 248 (1986) 267.

[22] G. Savard et al., Nucl. Inst. and Meth. B (2008), doi:10.1016/j.nimb.2008.05.091, in press.

[23] G. Savard et al., to be published.

[24] P. Dendooven et al., Nucl. Inst. and Meth. A 558 (2006) 580.

[25] G. Savard et al., Nucl. Inst. and Meth. B 204 (2003) 582. 